\title{
In vivo Three Photon Imaging of Neuronal Activities from Hippocampus in Intact Mouse Brain
}

Tianyu Wang ${ }^{1}$, Dimitre Ouzounov ${ }^{1}$, Nicholas G. Horton ${ }^{1}$, Jean C. Cruz Hernández ${ }^{2}$, Danielle Feng ${ }^{2}$, Nozomi Nishimura ${ }^{2}$, and Chris $\mathrm{Xu}^{1}$

1. School of Applied and Engineering Physics, Cornell University, Ithaca, NY, USA

2. Department of Biomedical Engineering, Cornell University, Ithaca, NY, USA

Optical imaging has enabled chronic observation of living mouse brain structure and function with single cell resolution [1]. Multiphoton microscopy (MPM) has significantly extended imaging depth to subcortical layers in highly scattering mouse brain [2-3]. Combined with genetically encoded calcium indicator GCaMP6s, we demonstrated that 3PM with $1350 \mathrm{~nm}$ excitation is capable of simultaneous recording of calcium transients in a neuron population in stratum pyramidal (SP) layer of cornus ammonis (CA1) region of hippocampus in intact mouse brain.

The excitation source for the custom-built multiphoton microscope was an optical parametric amplifier (OPA) operated at $1350 \mathrm{~nm}$ and a repetition rate of $250 \mathrm{kHz}$. After dispersion compensation, laser pulse width was $\sim 65 \mathrm{fs}$ after the objective. We imaged brain of mouse under anesthesia through cranial window two weeks after injection of AAV2/1 encapsulated GCaMP6s. The imaging site was approximately $2 \mathrm{~mm}$ posterior and $2.5 \mathrm{~mm}$ lateral to the bregma point.

With all overlaying cortical tissue intact, we imaged tissue structure in a stack from $700 \mu \mathrm{m}$ to $1250 \mu \mathrm{m}$ below brain surface. Fluorescence and third harmonic generation (THG) were simultaneously recorded. The THG signal delineates boundaries between deep cortical neurons, external capsule (EC), and hippocampus (Figure 1a). The maximum average power used to image SP layer in CA1 region of hippocampus was $\sim 36 \mathrm{~mW}$.

We further recorded calcium activities from 25 SP layer neurons simultaneously for 8 minutes (Figure 1b). Neuronal activities were continuously recorded with $128 \times 128$ pixel frames at a frame rate of 6.1 Hz. The time resolution was $164 \mathrm{~ms}$, which is adequate for GCaMP6s with a half decay time of $1.8 \mathrm{~s}$ for 10 action potentials (AP) [4]. Observed spike duration varies from $1 \mathrm{~s}$ to $20 \mathrm{~s}$. Relative fluorescence change $\left(\Delta \mathrm{F} / \mathrm{F}_{0}\right)$ of the spikes was as high as $700 \%$ (Figure 1c).

The method demonstrated here enables in vivo functional imaging of subcortical neuron population with single cell resolution within intact mouse brain. We anticipate that this technology will have an impact on minimally invasive investigation of neuronal circuit deep within a mouse brain.

\section{References}

[1] J. N. Kerr and W. Denk, Nature Reviews. Neuroscience 9 (2008), p. 195-205.

[2] N. G. Horton, et al., Nature Photonics 7, (2013), p. 205-209.

[3] D. A. Dombeck, et al., Nature Neuroscience 13 (2010), p. 1433-1440.

[4] D. S. Kim, et al., Neuroscience 2014 Short Course I: Advances in Multi-neuronal Monitoring of Brain Activity (2014) p. 12. 

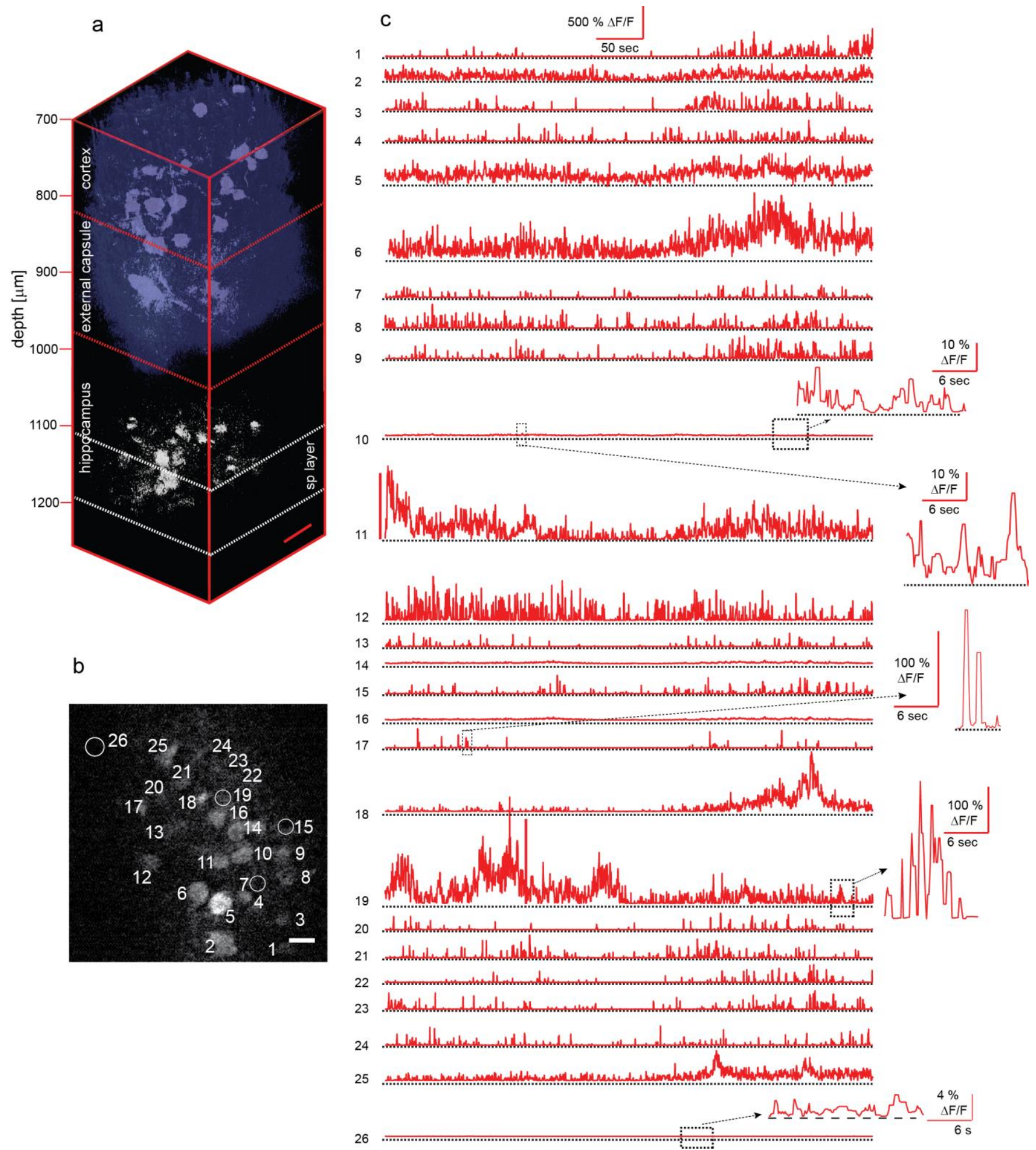

Figure 1. (a) Image stack of GCaMP6s labeled neurons spanning from cortex to hippocampus (fluorescence colored in bright grey, THG in dark purple, $175 \mu \mathrm{m}$ x $175 \mu \mathrm{m}$ field-of-view (FOV), taken with increments of $2 \mu \mathrm{m}$ in depth, scale bar $50 \mu \mathrm{m}$ ) (b) Neuronal population in the CA1 region of 1121$\mu \mathrm{m}$ depth (0.21 frames/s, 20 averages, 512x512 pixels, scale bar $20 \mu \mathrm{m})$ (c) Spontaneous $\mathrm{Ca}^{2+}$ transients recorded from the somata indicated in (b) 